\title{
Cathelicidins 家族抗菌肽研究进展
}

\author{
广慧娟 ${ }^{1}$ ，厉 政 ${ }^{1}$ ，王义鹏 ${ }^{2}$, 赖 仞 $^{3}$, 千海宁 ${ }^{1, *}$
}

(1. 大连理工大学 生命科学与技术学院, 辽宁 大连 116024; 2. 中国科学院烟台海岸带研究所 生物资源实验室, 山东 烟台 264003;

3. 中国科学院和云南省动物模型与人类疾病机理重点实验室, 中国科学院昆明动物研究所, 云南 昆明 650223)

摘要: Cathelicidins 是一类具有广谱抗微生物活性的多功能抗菌肽。迄今为止, 在几乎所有种类的脊椎动物体 内均有发现，在动物先天免疫系统中发挥极其重要的作用。Cathelicidins 不仅对普通革兰氏阳性菌、革兰氏阴性菌、 真菌以及病毒具有非常强的抗性，而且对许多临床分离而药菌株同样具有作用。Cathelicidins 具有特殊的杀菌机理， 不易产生耐药性。此外, cathelicidins 结构简单, 溶血活性和细胞毒性小, 因此极具开发潜力。该文主要对 cathelicidins 的结构与分类、生物活性与功能、作用特点与机制及其在医药领域中的应用前景和存在问题进行了综述。

关键词: Cathelicidins; 结构与功能; 抗菌活性; 耐药菌株

中图分类号: Q514.3; R96 文献标志码：A 文章编号：0254-5853-(2012)05-0523-04

\section{Progress in cathelicidins antimicrobial peptides research}

\author{
GUANG Hui-Juan ${ }^{1}$, LI Zheng ${ }^{1}$, WANG Yi-Peng ${ }^{2}$, LAI Ren ${ }^{3}$, YU Hai-Ning ${ }^{1, *}$
}

(1. School of Life Science and Biotechnology, Dalian University of Technology, Dalian 116024; 2. Biological Resources Laboratory, Yantai Institute of Coastal Zone Research, the Chinese Academy of Sciences, Yantai 264003, China; 3. Key Laboratory of Animal Models and Human Diseases Mechanisms of the Chinese Academy of Sciences and Yunnan Province, Kunming Institute of Zoology, the Chinese Academy of Sciences, Kunming 650223, China)

\begin{abstract}
Cathelicidins are a family of multi-functional antimicrobial peptides found in almost all types of vertebrates, where they play vital roles in the immune system. As they possess broad-spectrum antimicrobial properties, cathelicidins are not only strongly resistant to Gram-positive and Gram-negative bacteria, fungi, and viruses, but they are also active against many antibiotic-resistant clinical bacteria, adopting a special antimicrobial mechanism that is unlikely to lead to microbial resistance. Cathelicidins likewise possess simple structures, and low hemolytic and cytotoxic activities. Collectively, these features suggest potentially novel and exciting prospects for cathelicidins' application in medicine. Here, we review the structures, classification, activities, mechanisms, as well as prospective developments in the usage and application of cathelicidin antimicrobial peptides.
\end{abstract}

Key words: Cathelicidins; Structure and function; Antimicrobial activity; Resistant strains

近几十年, 由于抗生素的滥用导致的多药耐药 菌感染已经成为当代临床医学中急需解决的重大 难题。近来仅有三类新型抗生素(脂肽类、恶唑烷酮 类和链阳性菌素类)上市, 且均用于治疗 $\mathrm{G}^{+}$菌感染。 2010 年, 美国 FDA 仅批准了用于治疗急性细菌性 皮肤和皮肤结构感染(ABSSSI)及社区获得性细菌 性肺炎 $(\mathrm{CABP})$ 的头孢菌素类抗菌药—ceftaroline fosamil 注射剂。因此, 开发新型抗菌药物迫在眉睫。 而抗菌肽作为一种生物来源的、核糖体合成的天然 小分子多肽, 在新型抗菌药物开发领域所具有的潜
在优势已冊庸置疑(Hancock \& Sahl, 2006)。它们具 有广谱的抗菌活性, 对许多 $\mathrm{G}^{+}$菌和 $\mathrm{G}^{-}$菌, 以及真菌, 甚至病毒都有活性。此外, 由于它们的作用靶点主 要位于微生物的细胞膜上, 通过诱导微生物细胞膜 去极化，微生物细胞破裂而发挥作用，因此，不 易引起微生物抗性。而 cathelicidins 家族抗菌肽 作为脊椎动物来源的一个重要的抗菌肽家族, 具 有更加广谱高效的抗微生物活性、低溶血活性以及 细胞毒性, 在新型抗菌药物开发领域显示出巨大的 潜力。

收稿日期：2012-03-05; 接受日期：2012-08-15

基金项目：国家自然科学基金资助项目(41076098)

*通信作者(Corresponding author), E-mail: joannyu@live.cn 


\section{Cathelicidins 家族抗菌肽的一般特点}

\subsection{Cathelicidins 抗菌肽的结构和分类}

Cathelicidins 的前体由 N-端信号肽区域(29 30 个氨基酸残基，AA）、保守的中间 cathelin 区域 (94 114 个 AA)和高度特异的 $\mathrm{C}$-末端成熟肽区域 (12 100 个 AA)构成。Cathelin 区域的相对分子质量 大约为 11000 , 末端具有 4 个位置极度保守的 Cys 残基, 形成两对二硫键(C85 C96 和 C107 C124), 可能具有稳定 cathelicidins 分子结构的作用 (Sanchez et al, 2002)。Cathelin 区域富含带负电的 Asp 和 Glu 残基, 可能在前体肽的胞内运输和储存 过程中能够中和 C-末端带正电的成熟肽片段, 使前 体保持无活性状态, 从而避免对自身细胞的毒害 (Boman et al, 1994)。

按照二级结构可以大体分为四大类： $\alpha$-螺旋 cathelicidins、延伸-螺旋 cathelicidins、环状 cathelicidins 和 $\beta$-片层 cathelicidins。 $\alpha$-螺旋 cathelicidins：来源于 哺乳动物的 cathelicidins 抗菌肽大都属于 $\alpha$-螺旋构 型, 且一级结构一般只含有 23 40 个 AA。它们在 水溶液中大多是松散结构, 但是当与生物膜的相互 接触时会折叠成两亲性的螺旋结构(Turner et al, 1998; Tack et al, 2002)。目前，人、小鼠、兔、羊、 牛、猪、马、驴、鸡、狗、猫和金环蛇等许多物种 中发现的 cathelicidins 均属于 $\alpha$-螺旋型, 如 LL-37 (Agerberth et al, 1995)、BMAP-27、CAP-18 、 Pc-CATHs (Wang et al, 2011)、Cc-CATHs (Feng et al, 2011)、K9CATH、feCath 和 cathelicidin-BF (Wang et al，2008)等。延伸-螺旋 cathelicidins, 如 indolicidin 和富含 Pro 的抗菌肽类, 因含有特殊的氨基酸组成, 不易形成 $\alpha$-螺旋构型，而折叠成了更细长的结构。 包括牛的 Bac4、5、7 和 indolicidin (Scocchi et al, 1998), 来源于山羊的 Bac5, 猪的 prophenin-1、2 和 PR-39 (Pungerčar et al, 1993)等。

此外还有环状 cathelicidins, 牛嗜中性白细胞中 分离出的 dodecapeptide 和从绵羊骨髓细胞克隆的 同系物肽 Ovis. aires dodecapeptide (OaDode)。 $\beta$-片 层 cathelicidins，代表是来源于猪白细胞的 protegrins (PG1 5), 含有 16 18 个 AA, 它们通过二 硫键的稳定折叠成发卡结构。PG-1 的 NMR 谱表明 其结构是由一段 $\beta$-转角连接的两段反向平行 $\beta$-片层 结构, 形成中心的疏水区和两端亲水区的两亲性结 构(Kokryakov et al, 1993)。

\section{Cathelicidins 家族抗菌肽的活性与功能}

\subsection{Cathelicidins 家族抗菌肽的抗微生物活性}

2.1.1 $\alpha$-螺旋 cathelicidins $\alpha$-螺旋 cathelicidins 抗 菌肽对 $\mathrm{G}^{+}$和 $\mathrm{G}^{-}$菌均具有抗菌活性，而且包括一些 耐药性临床分离细菌，如耐甲氧西林金黄色葡萄球 菌、耐万古霉素粪肠球菌和多重耐药的铜绿假单狍 杆菌(Turner et al, 1998; Skerlavaj et al, 1996)。与人 $\alpha$-defensin 相比, LL-37 对包括铜绿假单狍杆菌和嗜 麦芽窄食单孢菌在内的许多细菌的抗菌活性更强 (Turner et al，1998)。小鼠腹腔注射 SMAP-29、 BMAP-27 及 BMAP-28 0.2 0.8 mg/kg，能够完全 保护由铜绿假单胞杆菌、金黄色葡萄球菌或大肠埃 希氏菌感染的腹膜炎小鼠，而对照组小鼠死亡率超 过 90\%(Mangoni et al, 1997)。来源于犬的 K9CATH, 对淋病奈瑟菌的抗菌能力比之前所发现其它 cathelicidin 家族抗菌肽高出 4 8 倍, 其 C 端高极性 的 7 个氨基酸残基可能导致病原微生物的降解并确 保内毒素的安全释放(Sang et al, 2007)。金环蛇来源 的 cathelicidin-BF 对多重耐药铜绿假单孢杆菌、多 重耐药肺炎克雷伯菌 08040724、耐药大肠杆菌和甲 型副伤寒沙门氏菌的 MIC 值分别为 $2.3 \mu \mathrm{g} / \mathrm{ml} 、 0.3$ $\mu \mathrm{g} / \mathrm{ml} 、 0.6 \mu \mathrm{g} / \mathrm{ml}$ 和 $1.2 \mu \mathrm{g} / \mathrm{ml}$ (Wang et al, 2008)。相 较哺乳动物来源的 $\alpha$-螺旋 cathelicidins，鸟类 cathelicidins 抗菌肽活性似乎更强。我们前期从环颈 雉体内发现的 Pc-CATHs, 即使在 $100 \mathrm{mM} \mathrm{NaCl}$ 的 条件下, 也展示出比氨芳青霉素、卡那霉素和人 LL-37 更强的抗菌能力, Pc-CATHs 对大多数金黄色 葡萄球菌的 $\mathrm{MIC}$ 低于 $1.48 \mu \mathrm{M}$ ，而后三者在 $2 \mathrm{mg} / \mathrm{ml}$ 浓度下检测不到抗菌活性。而且这种盐依赖的特性 也为治疗囊胞性纤维症和克罗恩病提供更多的选 择(Wang et al，2011)。而我们从鹤敦体内发现的 Cc-CATHs，虽然其抗菌活性略弱于鸡 fowlicidin-1 和 Pc-CATH1，但却具有极低的溶血性和细胞毒性 (Feng et al,2011)。

2.1.2 $\beta$-片层 cathelicidins PG 家族成员在体外不 仅表现出广谱的抗菌活性，而且还具有抗 HIV-1 病 毒的活性(Tamamura et al, 1995; Qu et al, 1996; Yasin et al, 1996; Lehrer \& Ganz, 1996)。在浓度为 $1 \sim 5 \mu \mathrm{g} / \mathrm{ml}$ 时, $\mathrm{PG}$ 家族成员能够杀死大量 $\mathrm{G}^{-}$菌包括 大肠杆菌、铜绿假单狍杆菌、肺炎克雷伯菌、鼠伤 寒沙门氏菌以及多种 $\mathrm{G}^{+}$菌如甲氧西林耐药金黄色 葡萄球菌、万古霉素耐药肠球菌和分支结核杆菌。 
此外 PG 对真菌和有包膜病毒也具有活性。PG 家族 抗菌肽在生理盐浓度溶液中或在血清存在情况下 仍具有活性(Lehrer \& Ganz, 1996)。

2.1.3 延伸-螺旋 cathelicidins 牛 Bac5 和 Bac7 是 高度阳离子化的多肽, 体外具有非常强的抗菌活性, 在 $0.5 \sim 20 \mu \mathrm{M}$ 浓度范围内, 能有效杀死大肠杆菌、 鼠伤寒沙门氏菌、肺炎克雷伯菌、表皮葡萄球菌和 巨大芽孢杆菌(Gennaro et al, 1989)。此外, 对螺旋体 也具有活性, 如钩端螺旋体和双曲螺旋体 (Scocchi et al, 1993)。对牛 Bac5 和 Bac7 抗菌机理研究表明, 这两种抗菌肽能够迅速增加敏感菌细胞内膜和外 膜的通透性, 导致细菌的内容物外泄, 此外还能抑 制细胞呼吸并降低细菌细胞内 ATP 水平。同样源于 牛的 indolicidin 抗菌谱非常广, 对 $\mathrm{G}^{+}$菌、 $\mathrm{G}^{-}$菌、真 菌, 甚至耐药的临床分离株(如铜绿假单胞菌、念珠 菌亚种和新型隐球菌的临床分离株) 以及寄生虫都 有很强的活性。另外, indolicidin 还具有抗 HIV-1 病毒活性和治疗小鼠系统曲霉病感染的功效。

2.1.4 环状 cathelicidins 天然 dodecapeptide 和 OaDode 对大肠埃希氏菌和金黄色葡萄球菌具有强大 的抗菌活性。研究结果表明, 当环状 dodecapeptide 分子解环成线性后, 它的抗菌活性就从抗 $\mathrm{G}^{-}$菌转变 为抗 $\mathrm{G}^{+}$菌。环状 dodecapeptide 与脂多糖的结合比 线性 dodecapeptide 更有效, 更能提高外膜的渗透 性(Romeo et al, 1988)。进一步研究表明, 环状 dodecapeptide 对鼠和人 $\mathrm{T}$ 淋巴细胞、鼠胚胎神经元 细胞和星形胶质细胞, 以及人成胶质细胞瘤细胞有 毒性。

\subsection{Cathelicidins 家族抗菌肽的其它生物学功能}

Cathelicidins 作为脊椎动物所特有的宿主防御 肽, 在脊椎动物抵抗外界微生物侵袭的天然免疫反应 过程中发挥了重要作用。除此之外, cathelicidins 还 具有如细胞趋化、伤口修复、抑制组织损伤、促进 血管生成等重要活性(Wuerth \& Hancock, 2011)。例 如 PR-39 通过抑制炎症反应中吞噬细胞 NADPH 氧 化酶活性, 直接减少超氧阴离子的产生, 从而减轻 局部组织的损伤(Shi et al，1996), 而且. PR-39 诱导 小鼠间质细胞表面硫酸类肝素蛋白多糖 syndecan-1 和 syndecan-4 的表达, 促进组织损伤的修复 (Shi et al, 1996)。另外, LL-37 通过结合并中和角质化细胞 中的细胞溶质 DNA, 阻断 AIM2(参与驱动炎症的 重要物质)炎性体激活, 减少牛皮痒炎症对机体的 损伤(Dombrowski et al, 2011)。
当机体受到 $\mathrm{G}^{-}$菌侵袭时，机体与细菌脂多糖 LPS 的相互作用可能会导致对机体有害的系统性反 应。体内实验研究表明, cathelicidins 可以结合内毒 素，对机体与 LPS 的相互作用具有调节功能 (Vandermeer et al, 1995)。此外, cathelicidins 还具有 调节适应性免疫的功能, 在对小鼠 cathelicidins 相 关的抗菌肽研究中第一次证明 cathelicidins 能够在 体内转变 $\mathrm{T}$ 细胞依赖的体液免疫活性, 因此能够调 节 $\mathrm{B}$ 淋巴细胞和 $\mathrm{T}$ 淋巴细胞的活性(Wuerth \& Hancock, 2011)。

\section{Cathelicidins 家族抗菌肽在医药领域的 应用前景和存在问题}

Cathelicidins 家族抗菌肽具有广谱的抗微生物 活性，对 $\mathrm{G}^{+}$菌、 $\mathrm{G}^{-}$菌、真菌、霉菌、原虫和部分有 包膜病毒均有活性。与其它家族，如 defensins、 hepcidins、昆虫抗菌肽和蛙科皮肤来源抗菌肽相比, cathelicidins 家族抗菌肽抗菌活性更强，最小抑菌浓 度(MICs)值可以达到几个 $\mu \mathrm{M}$ 水平(Wang et al, 2011; Feng et al, 2011)。此外, cathelicidins 家族抗菌肽杀 菌作用迅速，对金环蛇来源的 cathelicidin-BF 杀菌 动力学实验表明, 其在 $1 \mathrm{~min}$ 内即可杀死受试细菌, 比第三代号称“最强抗生素”的亚胺培南要快的多 (Wang et al, 2008)。更重要的是, 部分 cathelicidins 家族抗菌肽对大量临床分离耐药菌株，甚至是超级 耐药菌，具有非常强的活性，如 LL-37 对耐甲氧西 林金黄色葡萄球菌、耐万古霉素粪肠球菌和多重耐 药的铜绿假单狍杆菌均具有极强的杀菌活性 (Turner et al, 1998)。

目前 cathelicidins 家族抗菌肽药物研究热点主 要集中在消炎、抗感染和抗真菌等方面，应用方式 可以是局部的，也可以是系统的，剂型可以是口服， 也可以外用，如亳剂、喷雾剂、漱剂或洗液等。尤 其由于阳离子抗菌肽的表达与皮炎、侵入性烧伤脓 血症、肿瘤病毒引起的肉赘等之间的病理联系，使 得 cathelicidins 家族抗菌肽在这些疾病的局部治疗 方面有较好的开发前景。例如中科院昆明动物所优 选出蛇 cathelicidin 抗菌肽对 500 多株临床耐药菌株 显示了较强的抗菌活性, 同时具有极低的哺乳动物 细胞毒性以及溶血活性，优于美国正进行 III 期临 床的同类候选药物 pexiganan; 猪 cathelicidin 家族抗 菌肽— protegrins 治疗由放、化疗引起的口腔黏 膜炎，已进入临床 III 期试验; LL-37 作为治疗囊肿 
性纤维化支气管炎的局部杀菌剂及人抗菌肽 CAP-18 及其衍生物用于治疗上呼吸道感染、呼吸 道感染、鼻窦炎、中耳炎等疾病的药物均已经成为 生物制药领域内的研究热点。不仅限于医药领域, 在农业、畜牧业和日化用品领域, cathelicidins 也存 在巨大的应用潜能。

然而, cathelicidins 家族抗菌肽要开发为药物, 还面临几个亟待解决的问题: (1) cathelicidins 家族 抗菌肽生产上的限制，由于其相对分子质量小，易 被宿主细胞分解, 且对宿主细胞有一定毒性, 氨基 酸组成不均衡, 表达效率较低, 常以融合蛋白的形 式表达, 因此, 需要构建基因工程菌提高其表达量, 进一步改进分离和纯化技术; (2)安全性, 大多数

\section{参考文献:}

Agerberth B, Gunne H, Odeberg J, Kogner P, Boman HG, Gudmundsson GH. 1995. FALL-39, a putative human peptide antibiotic, is cysteine-free and expressed in bone marrow and testis [J]. Proc Natl Acad Sci USA, 92(1): 195-199.

Boman H, Marsh J, Goode JA. 1994. Antimicrobial Peptides [M]. London: John Wiley \& Son Ltd.

Dombrowski Y, Peric M, Koglin S, Kammerbauer C, Göss C, Anz D, Simanski M, Gläser R, Harder J, Hornung V, Gallo RL, Ruzicka T, Besch R, Schauber J. 2011. Cytosolic DNA triggers inflammasome activation in keratinocytes in psoriatic lesions [J]. Sci Transl Med, 3(82): $82 \mathrm{ra} 38$.

Feng FF, Chen C, Zhu WJ, He WY, Guang HJ, Li Z, Wang D, Liu JZ, Chen M, Wang YP, Yu HN. 2011. Gene cloning, expression and characterization of avian cathelicidin orthologs, Cc-CATHs, from Coturnix coturnix [J]. FEBS J, 278(9): 1573-1584.

Gennaro R, Skerlavaj B, Romeo D. 1989. Purification, composition, and activity of two bactenecins, antibacterial peptides of bovine neutrophils [J]. Infect Immun, 57(10): 3142-3146.

Hancock REW, Sahl HG. 2006. Antimicrobial and host-defense peptides as new anti-infective therapeutic strategies [J]. Nat Biotechnol, 24(12): 1551-1557.

Kokryakov VN, Harwig SSL, Panyutich EA, Shevchenko AA, Aleshina GM, Shamova OV, Korneva HA, Lehrer RI. 1993. Protegrins: leukocyte antimicrobial peptides that combine features of corticostatic defensins and tachyplesins [J]. FEBS Lett, 327(2): 231-236.

Lehrer RI, Ganz T. 1996. Endogenous vertebrate antibiotics [J]. Ann NY Acad Sci, 797(1): 228-239.

Mangoni ME, Aumelas A, Charnet P, Roumestand C, Chiche L, Despaux E, Grassy G, Calas B, Chavanieu A. 1996. Change in membrane permeability induced by protegrin 1 : implication of disulphide bridges for pore formation [J]. FEBS Lett, 383(1/2): 93-98.

Pungerčar J, Štrukelj B, Kopitar G, Renko M, Lenarčič B, Gubenšek F, Turk V. 1993. Molecular cloning of a putative homolog of proline/argininerich antibacterial peptides from porcine bone marrow [J]. FEBS Lett, 336(2): 284-288.

Qu XD, Harwig SS, Oren AM, Shafer WM, Lehrer RI. 1996. Susceptibility of Neisseria gonorrhoeae to protegrins [J]. Infect Immun, 64(4): 1240-1245.

Romeo D, Skerlavaj B, Bolognesi M, Gennaro R. 1988. Structure and bactericidal activity of an antibiotic dodecapeptide purified from bovine neutrophils [J]. J Biol Chem, 263(20): 9573-9575.

Sanchez JF, Wojcik F, Yang YS, Strub MP, Strub JM, Van Dorsselaer A, Martin M, Lehrer R, Ganz T, Chavanieu A, Galas B, Aumelas A. 2002. cathelicidins 家族抗菌肽仍然具有一定的溶血活性 和细胞毒性，而且目前的资料不足以证明 cathelicidins 家族抗菌肽长期使用后病原菌不会产 生耐药性; (3)稳定性, cathelicidins 家族抗菌肽由于 含有较多的碱性氨基酸残基，因此，易于被人体内 的蛋白酶降解，需要对其进行分子改造，在不影响 其抗菌活性的基础上，提高其稳定性。

目前, cathelicidins 家族抗菌肽的结构性质、生 物学活性与功能、作用机制及构效关系等的研究已 取得了显著的进展。相信 cathelicidins 家族抗菌肽 及其相关衍生物在药理学、毒理学、药代动力学、 临床疗效等方面的研究会有更快的发展，以解决越 来越严重的微生物耐药问题, 更好地造福于人类。

Overexpression and structural study of the cathelicidin motif of the protegrin-3 precursor [J]. Biochemistry, 41(1): 21-30.

Sang YM, Ortega MT, Rune K, Xiau W, Zhang GL, Soulages JL, Lushington GH, Fang JW, Williams TD, Blecha F, Melgarejo T. 2007. Canine cathelicidin (K9CATH): gene cloning, expression, and biochemical activity of a novel pro-myeloid antimicrobial peptide [J]. Dev Comp Immunol, 31(12): 1278-1296.

Scocchi M, Romeo D, Cinco M. 1993. Antimicrobial activity of two bactenecins against spirochetes [J]. Infect Immun, 61(7): 3081-3083.

Scocchi M, Wang SL, Gennaro R, Zanetti M. 1998. Cloning and analysis of a transcript derived from two contiguous genes of the cathelicidin family [J]. Biochim Biophys Acta, 1398(3): 393-396.

Shi J, Ross CR, Leto TL, Blecha F. 1996. PR-39, a proline-rich antibacterial peptide that inhibits phagocyte NADPH oxidase activity by binding to Src homology 3 domains of p47 phox [J]. Proc Natl Acad Sci USA, 93(12): 6014-6018.

Tack BF, Sawai MV, Kearney WR, Robertson AD, Sherman MA, Wang W, Hong T, Boo LM, Wu HY, Waring AJ, Lehrer RI. 2002. SMAP - 29 has two LPS - binding sites and a central hinge [J]. Eur J Biochem, 269(4): 1181-1189.

Tamamura H, Murakami T, Horiuchi S, Sugihara K, Otaka A, Takada W, Ibuka T, Waki M, Yamamoto N, Fujii N. 1995. Synthesis of protegrinrelated peptides and their antibacterial and anti-human immunodeficiency virus activity [J]. Chem Pharm Bull, 43(5): 853-858.

Turner J, Cho Y, Dinh NN, Waring AJ, Lehrer RI. 1998. Activities of LL-37, a cathelin-associated antimicrobial peptide of human neutrophils [J]. Antimicrob Agents Chemother, 42(9): 2206-2214.

Vandermeer T J, Menconi M J, Zhuang J, Wang HL, Murtaugh R, Bouza C, Stevens P, Fink M P. 1995. Protective effects of a novel 32-amino acid C-terminal fragment of CAP18 in endotoxemic pigs [J]. Surgery, 117(6): 656-662.

Wang YP, Hong J, Liu XH, Yang HL, Liu R, Wu J, Wang AL, Lin DH, Lai R. 2008. Snake cathelicidin from Bungarus fasciatus is a potent peptide antibiotics [J]. PloS One, 3(9): e3217.

Wang YP, Lu ZK, Feng Ff, Zhu W, Guang HJ, Liu JZ, He WY, Chi LL, Li Z, Yu HN. 2011. Molecular cloning and characterization of novel cathelicidin-derived myeloid antimicrobial peptide from Phasianus colchicus [J]. Dev Comp Immunol, 35(3): 314-322.

Wuerth K, Hancock REW. 2011. New insights into cathelicidin modulation of adaptive immunity [J]. Eur J Immunol, 41(10): 2817-2819.

Yasin B, Harwig S, Lehrer RI, Wagar EA. 1996. Susceptibility of Chlamydia trachomatis to protegrins and defensins [J]. Infect Immun, 64(3): 709-713. 